\title{
MULTILINGUAL OPEN-CONTENT SIGNAL PROCESSING LABORATORIES IN CONNEXIONS
}

\author{
Patrick Frantz, Richard Baraniuk* ${ }^{*}$ Hyeokho Choi* and Douglas L. Jones ${ }^{\dagger}$ \\ *Rice University, Department of ECE, \{jpfrantz,richb,choi $\} @$ rice.edu \\ †University of Illinois - Urbana-Champaign, Department of ECE, jones@ifp.uiuc.edu
}

\begin{abstract}
Due to inherent factors like a small and fragmented market and rapid hardware obsolescence, the conventional textbook is inadequate for DSP laboratory education. Freely available open-content materials that enable and promote both local customization and further development by a community of educators offer a fresh approach to lab text development that can surmount these barriers. In this paper, we overview a joint effort under the aegis of the Connexions Project to develop a large pool of DSP lab modules sufficient to serve as a complete, stand-alone text for several types of DSP lab courses. In addition, we introduce a pilot project whose aim is to demonstrate the suitability of using Connexions for languages other than English, such as the Japanese, Chinese and Thai languages.
\end{abstract}

\section{INTRODUCTION}

There is great need for information technologies to develop and deliver quality, up-to-date educational materials that show knowledge as a dynamic continuum, stretching across disciplines, languages and cultures. Ideally, many authors contribute to a knowledge repository from which instructors and/or learners create course texts. The resulting "book" should freely reference ideas and applications arising from both related and diverse fields. Presentation of material should not greatly influence knowledge storage methods, so that present and future display technologies are supported.

In addition, engineering disciplines are becoming increasingly globalized. Though English has become a dominant language for engineering education and a de facto standard for engineering conferences, publications, etc..., we should still recognize that students worldwide - especially below the graduate level - will certainly need some content in their native language. Any new content creation and deployment system should seamlessly support multiple languages.

Connexions is a new approach to authoring, teaching, and learning that aims to fully exploit modern information technology. Available free of charge to anyone under opencontent and open-source licenses, Connexions offers customtailored, current course material, is adaptable to a wide range of learning styles, and encourages students to explore the links among courses and disciplines. In contrast to the traditional process of textbook writing and publishing, Connexions fosters world-wide, cross-institution communities of authors, instructors, and students, who collaboratively and dynamically fashion " "modules" from which courses are constructed. We believe the ideas and philosophy embodied by Connexions have the potential to change the very nature of textbook writing and publishing, producing a dynamic, interconnected educational environment that is pedagogically sound, both time and cost efficient, and fun.

This paper overviews the philosophy and technology behind Connexions and describes its use in the building of digi-

0-7803-8560-8/04/\$20.00@2004IEEE tal signal processing (DSP) labs, a task for which it is particularly well-suited. A pilot project for internationalization is also described, showing that Connexions can readily support non-English content for truly global engineering education.

It should be noted that while this paper primarily discusses the use of Connexions for DSP education, it is an extremely flexible tool that can be used for course-building in any field, such as music, bioinformatics and psychology.

\section{THE CONNEXIONS PROJECT FOR DSP EDUCATION}

Several characteristics of digital signal processing (DSP) lab courses make them difficult to serve with conventional commercial textbooks. First, the market is fairly small, thus precluding a major investment by authors and publishers in the hope of a substantial commercial success. Second, the market is very fragmented; different lab equipment or DSP processors are used at each educational institution, and the course level, structure, and content may differ greatly, thus necessitating different textbooks or versions to serve an already small market. Essential materials generally include tutorials on using the particular lab setup and equipment and example code or wrappers that are site-specific. Third, DSP hardware changes rapidly, while the development and publication of a printed textbook takes quite some time, making texts nearly obsolete at the time of publication. For these reasons, conventional commercial textbooks and publishers are unlikely to ever serve DSP lab courses effectively.

While the limitations of conventional textbooks preclude them from effectively serving DSP lab courses, the situation is not hopeless. Most courses share many commonalities; for example, almost every DSP lab course covers FIR and IIR filter implementation. However, certain details, such as the assembly language instructions for a particular microprocessor, differ between platforms and preclude the use of a common printed text. Relatively minor modifications of the materials could effectively exploit the commonality of the bulk of the material but are currently not possible due to potential copyright violations and the fixed print medium.

The open-source software movement (e.g. Linux and GNU) suggests a new paradigm that may overcome these limits. In this spirit, we have undertaken a major communitybased project to develop open instructional materials for DSP lab education within the framework of Connexions.

\section{OVERVIEW OF THE CONNEXIONS PROJECT}

Connexions (see $[1,2]$ and cnx.rice.edu for more information) is a collaborative, community-driven approach to authoring, teaching, and learning that aims both to convey the dynamic continuum of knowledge and to ease and speed the course and curriculum development process. Launched in 1999 at Rice University, its hallmarks include:

- A Content Commons of diverse educational material spanning the knowledge continuum, modular for easy reuse, freely available to anyone worldwide, and hypertext linked; 


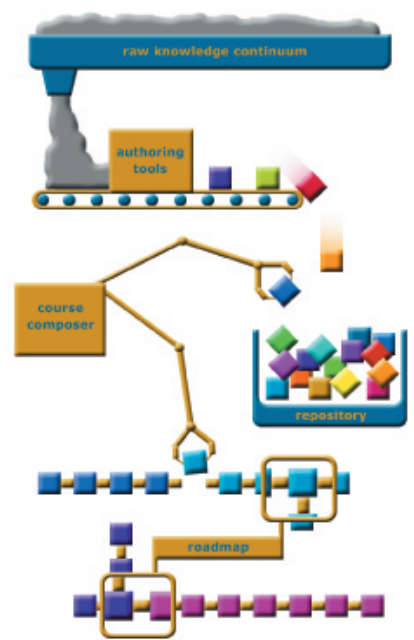

Fig. 1. The Connexions Course Factory

- Multilingual support so materials can appear simultaneously in many languages. Content already supports Japanese, Chinese and Thai, as well as several western languages;

- Free, open-source tools to aid visualizing and navigating the "connexions" among concepts, courses, and curricula;

- High-quality materials, with an iterative development process and an inherent quality assessment mechanism;

- Rapid, collaborative authoring of the materials by global communities of authors;

- Flexible, dynamic construction of an infinite variety of customized courses and curricula, using a coherent format (XML) and delivered in various forms, from web pages to ebooks to paper texts.

Connexions can be viewed as a kind of "course factory," as shown in Figure 1. A global community of authors, using special authoring tools, continually converts "raw knowledge" into small, self-contained modules of information and places them in the Content Commons repository to be used, re-used, updated, and adapted. (Modules can be thought of as special web pages that can contain hyperlinks, text, equations, applets, simulations, videos, and other multimedia elements.) Instructors use a Course Composer software tool to weave modules into customized courses. Students and other learners view modules and courses using special visualization and navigational tools designed to highlight the non-linear connexions among concepts.

The Connexions architecture and software toolkit consists of an XML language for content markup (including mathML for mathematics), a database repository to store and retrieve the modules, the Course Composer to build courses, an annotation capability for instructors to customize modules and for students to personalize them, a Roadmap browser to navigate courses and modules, and a printing tool that converts a course into a ready-to-print pdf text complete with table of contents and index. An integrated XML/mathML document editor is planned for the near future, as are wizards to help convert from LTEX, Microsoft Word, and other formats into XML. On the web, Connexions modules are viewable with a range of browsers, including Mozilla, Netscape 6 and above, and Microsoft Internet Explorer. The Roadmap navigator currently prefers Mozilla and Netscape. Last but not least, the Connexions author website enables groups of authors to form ad hoc workgroups to collaboratively develop new modules and courses. All Connexions software and tools are distributed open-source and free-of-charge. The first released version is in live use by faculty at Rice, UIUC, Ohio State (OSU), and a number of other institutions.
Rather than the traditional one-author-to-one-textbook content development model, Connexions links worldwide communities of authors to collaboratively create, expand, revise, and maintain the Content Commons. All materials are freely available under a Creative Commons open-content license analogous to the general public license (GPL) used for GNU and Linux software (www.creativecommons.org) Anyone can copy, modify, and redistribute modules, most even for commercial use. The result is a dynamic, up-to-date content base that makes the latest knowledge globally available.

Modularity and open-content development substantially lowers the barrier to entry into the author community. Most faculty currently do not write textbooks due the large time commitment required. Since in Connexions authors can now contribute a high-quality, high-impact module in an short time, many more college faculty, professionals, K-12 teachers, and even talented students can contribute materials.

In August 2004, Connexions hosts over 2,150 public modules and is used as the primary text in many courses. A particular content focus is DSP, manifested in two projects: graduate DSP theory materials [1], and DSP labs.

\section{THE CASE FOR CONNEXIONS DSP LAB TEXTS}

Connexions provides a near-ideal venue for developing DSP lab texts (or indeed any type of lab text). The low volume market has never been of great commercial interest, so the free, open-content model breaks the low-volume/high-cost barrier arising in print-based commercial publishing. The variety of different processors and lab setups makes it nearly impossible for a single printed text to address the needs of most laboratories, but the natural division of such courses into theory- and application-oriented concepts allows for the reuse of most materials across lab courses, with the exception of only the most platform-specific topics.

For example (see Figure 2), an assignment revolving around FIR filtering will typically involve a theory section on the concept, simulation exercises (in an environment such as Matlab), discussions of generic hardware theory (fixed-point arithmetic, for example) and specific hardware theory (FIR filter algorithms in Texas Instruments TMS320C54x assembly language, for example), and finally a section discussing the implementation and testing that are specific to a particular lab setup. The theory modules are readily available in Connexions and have most likely been seen by the students in their other DSP courses if those are Connexions-based. In fact, using already familiar DSP theory modules as a starting point will allow the students to not only establish greater continuity with their other course work but also begin the lab from comfortable territory. Simulation modules can be found both in DSP theory courses and other lab courses (even those involving just Matlab exercises). Similarly, hardware theory modules can be found in related courses.

Thus, in a fully developed Connexions environment, an instructor wishing to build a DSP lab class need only choose and assemble a set of pre-existing modules to craft the majority of her class. The remainder of the class can then be written in module form to cover the actual implementation issues of the local hardware platform. Even at this stage, she can potentially leverage modules provided by the equipment manufacturer (such as modules covering the assembly language instruction set for the microprocessor being used). A handful of segue modules (either written by the instructor or borrowed from similar DSP labs) tying theory to implementation can round out the course. This, of course, represents the minimum amount of effort required to craft a quality DSP lab. The instructor is free, under the open-content license, to customize and improve existing modules as she sees fit. These, in turn, may gain popularity and become the material most often used in other DSP labs, as commonly occurs in 


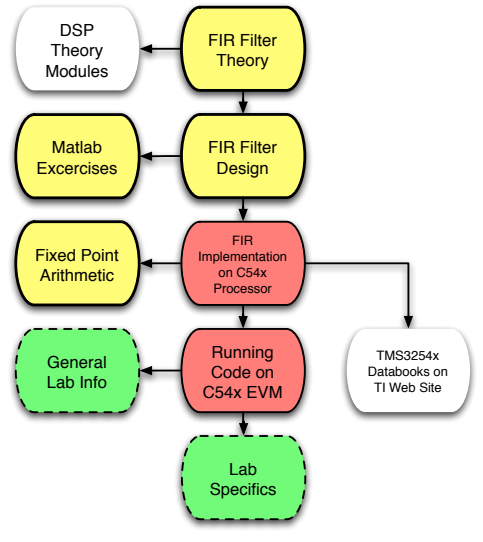

Fig. 2. An example of how an FIR filtering lab can be constructed using Connexions.. Each box represents a module, and arrows represent links between modules. Boxes with thick boundaries indicate DSP theory and exercise modules that can be used at other institutions with little modification. Boxes with thin boundaries are processor-specific. Boxes with dashed boundaries are specific to a particular lab setup.

such an open-source development environment.

In short, though instructors may lack the time, resources, or desire to independently develop a complete text of lab materials, they can still fashion, with relatively little effort, a comprehensive DSP lab text that accommodates the hardware available at their respective institutions. By leveraging pre-existing materials and perhaps adding a module or two on topics of special interest and expertise, an instructor can create and share a full lab text with little more effort than that needed for preparing supplementary handouts with assignments and site-specific information.

\section{CONNEXIONS DSP LABS}

In 2001 we realized that Connexions could provide a new and better mechanism for sharing DSP lab material and for enabling a community of authors to collaboratively develop a common instructional resource of ever greater breadth and utility. Former UIUC ECE 320 teaching assistants, instructors and the Connexions staff at Rice developed a complete set of lab modules in Connexions based on original ECE 320 material. In fact, two versions of ECE 320 were developed, one all-assembly and one assembly-with-C-compiler.

To confirm the capability of the system and the efficacy and completeness of the materials, ECE 320 (physically located in Urbana, Illinois) has been taught since the Fall 2002 semester using solely Connexions-based materials located on the Connexions server (physically located at Rice University in Houston, Texas)

To augment the UIUC ECE 320 materials for Rice ELEC $226,424 / 427$, and 434 , a series of modules are being developed to cover both basic DSP theory and TMS320C6211-, TMS320F2812- and MSP430F169-based algorithm implementations. Figure 2 illustrates an example FIR filtering lab developed using Connexions modules. In the figure, each box represents a different module, which were either obtained from other Connexions courses (for example, Rice ELEC 301, Signals and Systems) or written from scratch. The boxes with thick boundaries indicate the DSP theory and exercise modules that can be used at other institutions with little modification, since they are common to any FIR filtering lab. The boxes with thin boundaries are the processorspecific parts of the lab. For example, one can replace them with modules developed for the TMS320C6713 processor if floating-point-based course is desired. The boxes with dashed boundaries are specific to a given lab setup and would need to be replaced with site-specific lab setup information.

\section{USING THE CONNEXIONS DSP LABS}

The Connexions DSP lab materials build on over fourteen years of DSP lab instruction and over ten years of collaborative development of instructional lab materials at UIUC, Rice, and OSU. Much of the content has evolved in tandem with the UIUC ECE 320 course, and to a large extent reflects its goals and structure. With the addition of the Rice ELEC 226, 424/427 and 434 modules, the material is suited for a variety of course organizations, including

- a semester-long project-oriented DSP lab,

- a quarter- or semester-long DSP lab,

- a hands-on lab supplement as part of a DSP theory course,

- a self-study course in real-time DSP implementation

Being highly modular, the connexions modules can be easily modified and regrouped to develop texts to serve various different goals. A course emphasizing DSP techniques might forgo a major project and instead use the supplementary modules to complete a quarter or semester of weekly lab assignments. A one-hour hands-on lab supplement to a signal-processing lecture course could stretch the first few units (through spectral analysis, for example) over a semester, thereby reinforcing and enhancing students' understanding of the core signal processing theory and algorithms. Due to the self-paced, tutorial design of the materials, a student can independently learn the aspects of real-time DSP implementation that interest them, for example as preparation for an independent design project.

The materials and assignments from UIUC, Rice, OSU and others reflect our belief that a thorough instruction in signal processing implementation requires exposure to assembly-language programming of fixed-point DSP microprocessors, as this represents an important component of current and at least near-future industrial practice. Instructors with other goals or perspectives may find most of the tutorial, design material, and assignments relevant even if they choose compiler-based or non-real-time implementation.

Laboratories using different development systems or different DSP microprocessors will likely find the material well suited for their needs; only the hardware-specific instructions need be modified. By choosing different sets of Connexions modules and modifying and rewriting the hardwarespecific ones, many different lab texts supporting different lab setups can be easily generated with minimal effort. In this manner, several courses have been established at Rice using common content, but based on different Texas Instruments processors and with different course goals: ELEC226 (MSP430F169), ELEC 424/427 (TMS320F2812), ELEC 434 (TMS320C6211).

\section{MULTILINGUAL DSP LAB DEMO}

Recognizing that engineering and education are international disciplines, we began a pilot project to globalize Connexions by introducing DSP content in other languages: Japanese, Chinese and Thai, in particular. To complete this demonstration, we began by translating existing Connexions modules used in Doug Jones' ECE 320 DSP lab at UIUC. This existing content provided a stable base from which to work, since it has been in active use for several years.

A subset of the modules in ECE 320 have already been translated, as shown in Figures 3 (Japanese), 4 (Chinese) and 5 (Thai). These and other translated modules can be viewed via the Connexions web site by loading the course "DSP Laboratory with TI TMS320C54x (International Demo)." While Connexions does support multiple browsers and platforms, the international demonstration is best viewed using the Mozilla browser with the Connexions 


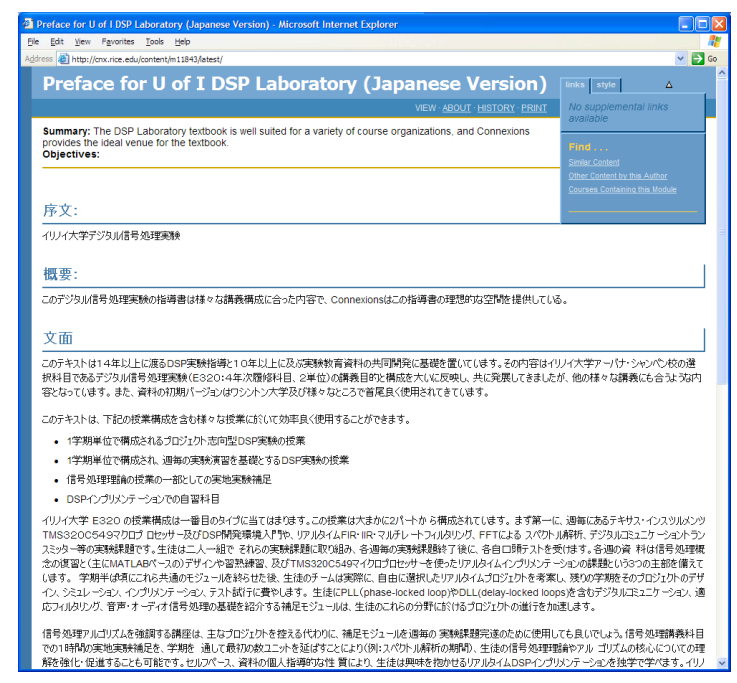

Fig. 3. Sample Japanese Content

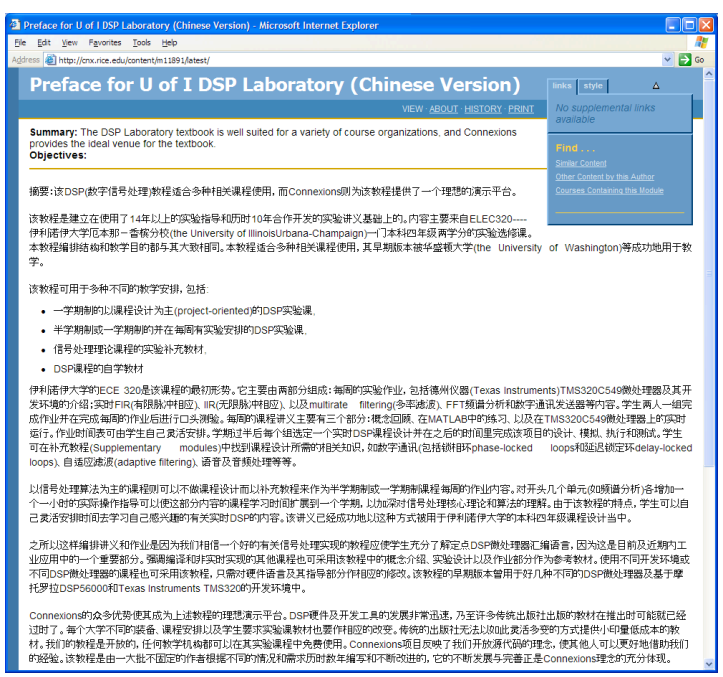

Fig. 4. Sample Chinese Content

Roadmap plugin and MathML fonts, or Internet Explorer with the MathPlayer plugin (the Connexions Roadmap plugin does not currently support IE). For more information on browser support and instructions on use of these tools, please visit the Connexions web site at $\mathrm{cnx}$.rice.edu.

The demonstration clearly shows that Connexions can support multilingual content. Its support for content in the Unicode text standard allows even complex East and Southeast Asian fonts to be displayed without problem, as can be seen in the screenshots here and on the Connexions web site.

However, to make Connexions truly global, there are a few areas of improvement that will make multilingual support even better. First, printing modules (to PDF) with Asian fonts is only partly functional. Second, most users will want to see a Connexions interface (e.g. the Roadmap plugin) in their own language. The interface is currently in English, but an effort is underway to make it available in other languages. Nevertheless, neither of these factors are major obstacle to complete internationalization of Connexions.

\section{CONCLUSIONS}

Connexions is an experimental, open-source/open-content initiative that offers an alternative way to create, maintain and use textbooks and learning materials. By design, Connexions greatly impacts current teaching and learning modes and the development and sharing of knowledge. Combined with

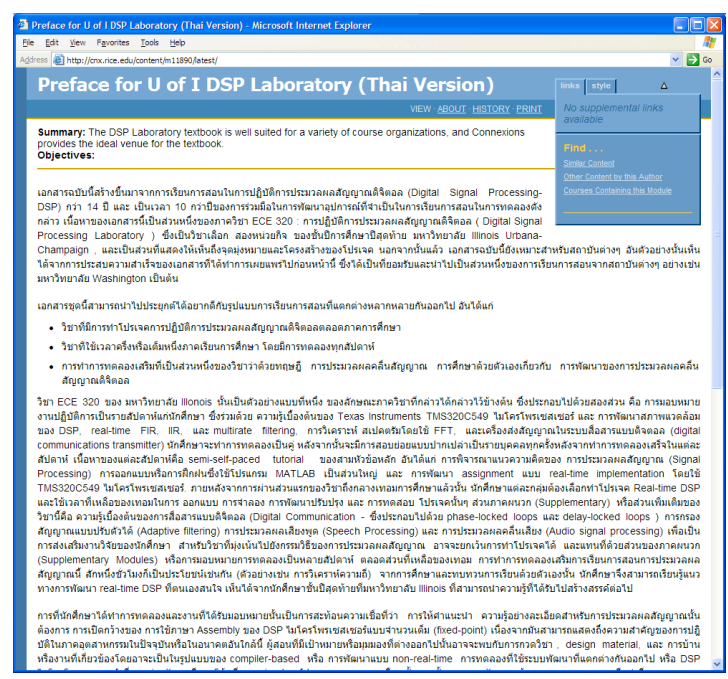

Fig. 5. Sample Thai Content

powerful software tools, Connexions gives free access to educational materials that can be readily manipulated to suit their individual learning styles as they explore links among concepts. The free tools also foster the development, manipulation, and continuous refinement of educational material by diverse communities of authors and instructors.

The hard work of the participants in this project has already resulted in a large pool of DSP lab modules sufficient to serve as the complete, stand-alone text for several types of DSP lab courses. In this paper, we have shown how Connexions is extremely well suited for DSP laboratory content generation, and we have also demonstrated that Connexions can also be used for content in a variety of languages, specifically Japanese, Chinese and Thai.

Though Connexions was originally conceived as a tool for DSP content authoring and education, it is very suitable for use in other fields, as well. The Connexions materials are open-content licensed, so anyone is free to use, modify, and add to them. All of these courses and modules are available for free use and re-use at cnx.rice.edu.

\section{ACKNOWLEDGMENTS}

Thanks to the Hewlett Foundation, CLASS Foundation, and Rice University for supporting the development and launch of Connexions. Thanks to Texas Instruments and Motorola for the DSP hardware and software development tools. And thanks to the dedicated Connexions staff, including Ricky Radelli-Sanchez, Adan Galvan, and Brent Hendricks.

Thanks also to all of those who helped in the development of ECE 320 at UIUC. Special thanks to Emiko Yamai, Kanglin Wang and Kamolchanok Kriengchaipruck for their help with content translation.

\section{REFERENCES}

[1] R. Baraniuk, C. Burrus, B. Hendricks, D. Johnson, D. Jones, R. Nowak, J. Odegard, K. Ramchandran, R. Reedstrom, I. Selesnick, and W. Wilson, "Connexions: DSP Education for a Networked World," in IEEE International Conference on Acoustics, Speech, and Signal Processing (ICASSP), 2002.

[2] S. Appadwedula, R. Baraniuk, M. Berry, M. Butala, H. Choi, M. Huan, D. Jones, M. Kramer, D. Moussa, L. Potter, D. Sachs, B. Wade, and R. Wagner, "OpenContent Signal Processing Laboratories in Connexions," in IEEE International Conference on Acoustics, Speech, and Signal Processing (ICASSP), 2003. 